\title{
Congenital afibrinogenemia in a 4-year-old girl complicated with acute lymphoblastic leukemia
}

\author{
Alper Özcan ${ }^{\oplus}$, Bahadır Samur ${ }^{\oplus}$, Şefika Akyol $^{\odot}$, Necdet Arda Erdoğmuş ${ }^{\oplus}$, \\ Türkan Patıroğlu ${ }^{\odot}$, Musa Karakükçü ${ }^{\oplus}$, Ekrem Ünal $^{\odot}$ \\ Division of Pediatric Hematology Oncology, Department of Pediatrics, Erciyes University Faculty of Medicine, Kayseri, Turkey.
}

\begin{abstract}
Background. Congenital fibrinogen deficiency is one of the rare inherited coagulation disorders. Congenital fibrinogen deficiency complicated with a hematological malignancy can be life threatening.

Case. We present a four-year-old girl with congenital fibrinogen deficiency complicated with acute lymphoblastic leukemia.
\end{abstract}

Conclusion. This case aims to highlight therapeutic approaches for the management of afibrinogenemia patients with acute leukemia.

Key words: acute lymphoblastic leukemia, child, congenital afibrinogenemia.

Congenital afibrinogenemia (CA) is a rare autosomal recessive bleeding disorder that is characterized by the undetectable low level of fibrinogen. ${ }^{1} \mathrm{CA}$ has an estimated prevalence of one for 1,000,000. The most common clinical symptoms are mucocutaneous, softtissue, joint and genito-urinary spontaneous bleeding, traumatic, or surgical bleeding. ${ }^{2,3}$ Hemorrhagic diathesis and arterial and venous thromboembolic complications can develop in a patient with CA. ${ }^{4}$ CA complicated with a hematological malignancy can be life threatening. Acute lymphoblastic leukemia (ALL) is the most common cancer in children and represents the leading cause of cancerrelated mortality in children. Survival rate of ALL is approximately $85 \% .{ }^{5}$ Review of the literature has shown that the occurrence of

Ekrem Ünal

drekremunal @yahoo.com.tr

Received 24th May 2018, revised 11th July 2019, accepted 27th August 2019.

This paper was presented in 11th Annual Congress of the European Association for Haemophilia and Allied Disorders (EAHAD) 2018 in Madrid, on February 7-9, 2018 (Poster presentation). hematological malignancies in patients with coagulation disorders are rare, with only some reported cases of leukemia in patients with haemophilia. ${ }^{6}$ However, afibrinogenemia with ALL has never been reported. The coincidence of these two diseases together is important which has led to challenges in developing treatment strategies. We describe a four-yearold girl with CA complicated with ALL.

\section{Case Report}

A 4-year-old girl with a history of CA admitted to the emergency department with a complaint of bleeding in her mouth. From her medical history it was learned that the patient was diagnosed with CA because of intracranial hemorrhage at the age of 1 day. Her 22-year-old brother was also diagnosed with CA.

Her physical examination at the time of admission disclosed petechial rash and ecchymoses, pallor, and hepatosplenomegaly. Vital signs were normal. The white blood cell count of the patient was $94,530 / \mathrm{mm}^{3}$ with $90 \%$ blasts, the measured hemoglobin level was 6,9 $\mathrm{g} / \mathrm{dl}$, and platelet count was $137,000 / \mathrm{mm}^{3}$. Her 
complete metabolic panel was normal except for an LDH of $440 \mathrm{u} / \mathrm{L}(135-225 \mathrm{u} / \mathrm{L})$. Peripheral smear examination showed presence of $90 \%$ blasts which were bigger than lymphocytes with high nuclear-cytoplasmic ratio, moderate amount of cytoplasm, round to oval nucleus, fine chromatin and 0-1 nucleoli. Red blood cells were predominantly normocytic normochromic. Platelets were reduced. Bone marrow examination showed $85 \% \mathrm{~L}_{1}$-type blasts which were smaller and twice the diameter of a red cell, high nuclear cytoplasmic ratio, regular shape of nucleus and some small nucleoli.

Immunophenotypical analyses showed that blasts were positive for CD19, CD10, CD38, CD58, CD79a, HLA-DR but were negative for myeloid markers. Flow cytometry findings were compatible with ALL. In addition, karyotypical analyses of peripheral blood, and bone marrow aspiration revealed no abnormalities. Cytogenetic analysis was normal. The cerebrospinal fluid examination showed no blasts. She received intensive chemotherapy for intermediate risk group ALL according to the Berlin-Frankfurt-Munich ALLIC 2009 protocol.

The Prothrombin time (PT) $>100 \quad(10-14$ second), activated partial thromboplastin time (aPTT)>120 (25-36 second), International Normalized Ratio (INR) $>10$ were prolonged; fibrinogen level was not detectable. D dimer $190(0-550 \mu \mathrm{g} / \mathrm{L})$, antithrombin III 99.7\% (83$118 \%$ ) levels were in normal ratio. Levelsof fibrinogen and platelets count were influenced by her malignancy and chemotherapeutic treatment. Fibrinogen replacement therapy was given before the invasive procedures such as bone marrow aspiration, lumbar puncture, catheter insertion and removal. The target level of fibrinogen was $100 \mathrm{mg} / \mathrm{dL}$. Thrombocyte suspension was given to the patient during active bleeding and before invasive procedures (platelet count $<50,000 / \mu \mathrm{L}$ ).

The bone marrow evaluations of $15^{\text {th }}-33^{\text {rd }}$ day were found to be in remission. As paradox arterial and venous thromboembolic complications can develop in patients with CA, low-molecular-weight heparin (enoxaparin sodium) injection, $1 \mathrm{mg} /$ day, was started for the prophylaxis of thromboembolic complications of CA during the chemotherapy regimen. During the treatment protocol the patient's fibrinogen levels, hemogram, PT, aPTT, antifactorXa tests were evaluated not just during symptomatic times of bleeding and before invasive proceduresandthey were also checked periodically 2 times each week.

Finally, the patient finished the induction, consolidation, re-induction, and the continuation parts of the ALLIC 2009 protocol without any complications related to CA. During follow-up laboratory results revealed thePT $>100$ (10-14 second), PTT $>150 \quad$ (25-36 second), INR $>5$ were prolonged; $\mathrm{D}$ dimer $300(0-550 \mu \mathrm{g} / \mathrm{L})$, antithrombin III 90\% (83$118 \%)$ levels were in normal ratio. Fibrinogen level was 99 (180-350 mg/dl). Fibrinogen level waselevated as the patient receivedfibrinojen concentrate before the catheter removal during the continuation therapy. Peripheral blood counts returned to normal she remained in clinical and laboratory remission without any bleeding diathesis symptoms or thrombosis. Written consent was obtained from the family for the publication of this case report.

\section{Discussion}

CA is a rare autosomal recessive disorder. Intracranial hemorrhage is a common site of bleeding in children with CA. CA may manifest as a neonatal intracranial hemorrhage due to traumatic delivery. ${ }^{1}$ There are some challenges in management of the patients with CA resulting from both bleeding and thromboembolic complications. ${ }^{2}$ This twofaced problem may be harder in patients with hematological malignancies. In the English and Turkish medical literature cases of acute childhood leukemia in a patient with hemophilia, von Willebrand syndrome were reported. To the best of our knowledge, this is the first reported case of ALL in patient with CA. It is important to show how to manage the 
issues with the bleeding complications related to $\mathrm{CA}$ and acute leukemia. ${ }^{3-6}$ In addition, the platelet numbers, and functions and factor levels that may also be affected by the ALL treatment. Thrombocytopenia can exacerbate the bleeding complications of CA; so when platelet transfusion is required the platelet count should be kept relatively higher than leukemic children without CA. Leukemic children with CA may require frequent platelet transfusions, and plasma derived fibrinogen concentrate. Children with CA complicated with acute leukemia may have long-term abnormalities such as inhibitor development from higher factor concentrate usage like hemophiliac patients. In addition the increased frequent of usage of blood products may result in increased adverse reactions including febrile reactions, bacterial \& viral contamination, transfusion related acute lung injury, etc. ${ }^{7-9}$ Thus, managing of bleeding disorders like afibrinogenemia/disfibrinogemia with ALL is extremely sophisticated.

The presented patient was regularly monitored with a detailed blood investigation before invasive procedures or in symptomatic bleeding times. As the half-life of fibrinogen is $2-4$ days, the patient was evaluated twice a week using PT, aPTT, antifactorXa and fibrinogen levels tests. As described by Peyvandi et al. ${ }^{10}$ fibrinogen replacement therapy was given before the invasive procedures such as bone marrow aspiration, lumbar puncture, catheter insertion and removal. The target level of fibrinogen was $100 \mathrm{mg} / \mathrm{dL}$. The critical level of transfusion for thrombocytopenia was detected according to Estcourt et al. ${ }^{11}$ Thrombocyte suspension was transfused during active bleeding and before invasive procedures (platelet count $<50,000 / \mu \mathrm{L}$ ). With this methodology, the clinical phenotype of the presented child was moderate, and spontaneous bleeding was very rarely observed.

Furthermore, children with CA (in addition to these classic clinical features of bleeding episodes) may present with increased risk of thrombosis which often necessitating the concurrent use of anticoagulants and fibrinogen. ${ }^{12}$ Because of our previous reported experience low-molecular-weight heparin, enoxaparin was started to balance the two sharp faces of the blade. It was speculated that, enoxaparin prophylaxis may be an effective way to control thrombosis which may result from CA or increased risk of hematologic malignancy such as catheter insertion, steroid and L-Asparaginase usage. ${ }^{13,14}$

Arterial and venous thromboembolic events have rarely been reported in patients with CA. But thrombosis is difficult to manage because of the bleeding tendency of the patients with CA. ${ }^{12}$ Amri et al. ${ }^{15}$ recommended the treatment of low-molecular weight heparin in patients with CA. In the experience of the presented case, we speculate that close monitoring, the usage of enoxaparin $1 \mathrm{mg} / \mathrm{kg} /$ day with fibrinogen replacement without any anti-agregant treatment seemed to be effective.

In conclusion, CA is known as a rare disease with different underlying disorders. To the best of our knowledge this is the first report of the co-occurrence of leukemia and CA in a pediatric patient. Thus, this strange togetherness led to a challenge in developing a treatment strategy and which may have resulted in bleeding complication. The togetherness of leukemia and bleeding disorders may require much more attention and the development of novel treatment strategies.

\section{REFERENCES}

1. Patiroglu T, Ozdemir MA, Unal E, et al. Intracranial hemorrhage in children with congenital factor deficiencies. Childs Nerv Syst 2011; 27: 1963-1966.

2. Ruiz-Sa'ez A. Thrombosis in rare bleeding disorders. Hematology 2012; 17(Supp 1): S156-S158.

3. Turner ML, Watson HG, Russell L, Langlands K, Ludlam CA, Parker AC. An HIV positive haemophiliac with acute lymphoblastic leukaemia successfully treated with intensive chemotherapy and syngeneic bone marrow transplantation. Bone Marrow Transplant 1992; 9: 387-389.

4. Kawakami K, Takezaki T, Nakazono S, et al. Acute childhood leukaemia in a patient with haemophilia: first report in Japan. Acta Paediatr Jpn 1994; 36: 9194. 
5. Kızmazoğlu D, Sarı S, EvimSezgin M, et al. Assessment of health-related quality of life in pediatric acute lymphoblastic leukemia survivors: perceptions of children, siblings, and parents. Turk J Haematol 2019; 36:112-116.

6. Dunn AL. Malignancy in patients with haemophilia: a review of the literature. Haemophilia 2010; 16: 427436.

7. Patiroglu T, Murataldı S, Koklu E, Karakukcu M. Pleural empyema caused by Salmonella typhimurium in a patient with acute lymphoblastic leukemia. Erciyes Med J 2003; 25: 204-207.

8. Sinha R, Sodhi K, John B, Singh D. A rare case of acute lymphoblastic leukaemia with hemophilia A. Ital J Pediatr 2009; 35:40.

9. Zhang L, Li H, Zhao H, Zhang X, Ji L, Yang R. Retrospective analysis of 1312 patients with haemophilia and related disorders in a single Chinese institute. Haemophilia 2003; 9: 696-702.

10. Peyvandi F, Haertel S, Knaub S, Mannucci PM. Incidence of bleeding symptoms in 100 patients with inherited afibrinogenemia or hypofibrinogenemia. J Thromb Haemost 2006; 4: 1634-1637.
11. Estcourt LJ, Malouf R, Doree C, Trivella M, Hopewell S, Birchall J. Prophylactic platelet transfusions prior to surgery for people with a low platelet count. Cochrane Database Syst Rev 2018; 9: CD012779.

12. Ozdemir MA, Işik B, Patiroglu $T$, et al. A case of congenital afibrinogenemia complicated with thromboembolic events that required repeated amputations. Blood Coagul Fibrinolysis 2015; 26: 354-356.

13. Kose D, Paksoy Y, Koksal Y, Unal E. Neurological complication of non Hodgkin lymphoma in childhood: experience from a single center in Turkey. Childs Nerv Syst 2014; 30: 639-645.

14. Unal E, Yazar A, Koksal Y, Caliskan U, Paksoy Y, Kalkan E. Cerebral venous sinus thrombosis in an adolescent with Ewing sarcoma. Childs Nerv Syst 2008; 24: 983-986.

15. Amri Y, Kallel C, Becheur M, et al. Hypodysfibrinogenemia: a novel abnormal fibrinogen associated with bleeding and thrombotic complications. Clin Chim Acta 2016; 460: 55-62. 